Cécile Delettres / Britta Jallerat-Jabs

\title{
„Ja müssen Sie jetzt nix irgendwie aufschreiben?" - Eine empirische Untersuchung zur Verwendung von irgendwie im gesprochenen Deutsch
}

\section{Einleitung}

Trotz seiner auffallenden Verwendungshäufigkeit im heutigen gesprochenen Deutsch wurde unseres Wissens das Lexem irgendwie bisher noch nicht systematisch auf seine Bedeutung bzw. Bedeutungsfacetten und seine Funktion(en) hin untersucht. Betrachtet man Nachschlagewerke oder Arbeiten, die irgendwie berücksichtigen, fällt die uneinheitliche klassifikatorische Zuordnung des Lexems ins Auge: So wird irgendwie zum Beispiel als „Indefinitadverb“ (Duden 2016: § 846), „modalisateur“ (deutsch: Modaladverb; Schanen/Confais 2001: § 821) oder „Heckenausdruck“ (Schwitalla 1997: 173; Papantoniou 2010) bezeichnet; Thaler (2008) ordnet irgendwie gleich zwei Kategorien zu, nämlich einerseits den „particules illocutoires“ (deutsch: Abtönungspartikeln: 199), andererseits auch den „modalisateurs adverbiaux“ (deutsch: Modaladverbien: 200f.). Günthner/König (2015) haben unlängst gezeigt, dass irgendwie unter bestimmten Voraussetzungen als „Diskurspartikel“ fungieren kann.

Diese Vielzahl an Benennungen bzw. Zuordnungen scheint auf Polyfunktionalität von irgendwie hinzudeuten. In der Tat befassen sich die genannten Arbeiten mit jeweils einer bestimmten Verwendungsweise des Lexems, wobei überhaupt nur bei Günthner/König sowie Papantoniou irgendwie im Mittelpunkt der Analyse steht. Günthner/König konzentrieren sich zudem auf eine besondere und unseren eigenen empirischen Daten zufolge seltene - syntaktische Position, nämlich auf irgendwie im Nachfeld.

Der vorliegenden Untersuchung, die sich in erster Linie als empirische Bestandsaufnahme versteht, liegt eine andere Vorgehensweise zugrunde. Wir haben 360 Vorkommen von irgendwie in einem mündlichen Korpus auf die Verschiedenheit ihrer semantischen bzw. pragmatischen Verwendungsmodalitäten hin betrachtet, in der Absicht, einen Beitrag zu einer umfassenderen Beschreibung des Lexems irgendwie zu leisten, die uns in Anbetracht seiner Gebrauchshäufigkeit in der gesprochenen Sprache aus linguistischer Sicht wünschenswert erscheint. Das untersuchte Korpus setzt sich aus 26 durchschnittlich einstündi- 
gen Leitfadeninterviews zusammen, die zwischen 2005 und 2010 mit deutschsprachigen Informant/innen in mehreren deutschen Großstädten ${ }^{1}$ durchgeführt wurden (siehe Jabs 2012). Die Interviews wurden auf einen Tonträger aufgenommen und anschließend verschriftlicht. Der schriftliche Korpusumfang beträgt 236.346 Wörter. Bei dieser Studie ging es ursprünglich um eine soziolinguistische Fragestellung zum Thema „Lebensstil und Sprachverhalten“, wobei der Lebensstil der Gewährspersonen, die unterschiedlichen sozialen Milieus entstammen, anhand der Indikatoren Familiensituation, berufliche Situation, Geschmacksrichtungen im Bereich des Wohnens, der Kleidung, der kulturellen- und Freizeitaktivitäten bestimmt werden sollte. Unsere Untersuchung zu den Verwendungsmodalitäten von irgendwie entspricht somit einer sekundären Verwertung des Korpus. Dieses bietet den Vorteil, relativ umfangreich zu sein und somit den Vergleich nicht nur zwischen 360 Vorkommen von irgendwie, sondern auch, unter Einbezug der Interviewerin, zwischen 27 Sprecher/innen unterschiedlichen Alters (zum Zeitpunkt der Interviews waren die Sprecher/innen zwischen 30 und 70 Jahre alt) sowie verschiedener sozialer und geografischer Herkunft zu ermöglichen. Wir verfügen über eine literarische Verschriftlichung des Sprachmaterials (nicht über eine phonetische Transkription). Phonetische Aspekte der Verwendung von irgendwie werden demnach hier nicht berücksichtigt.

\section{Verwendungsmodalitäten von irgendwie}

Aus der systematischen Auswertung der Korpusdaten hat sich die Differenzierung von fünf Verwendungstypen ergeben, die in den Abschnitten 2.1 bis 2.5 mittels für den jeweiligen Untertyp besonders charakteristischer Korpussequenzen veranschaulicht werden.

\subsection{Vagheitsindikator}

Gülich/Furchner (2002) bezeichnen irgendwie als „Vagheitsindikator“ (ebd.: 165) (siehe auch weiter unten: 2.2 Formulierungsschwierigkeiten). Zwar möchten wir diesen Begriff übernehmen, ihn aber, da er unseren Untersuchungen zufolge nicht alle Verwendungsweisen des Lexems in unserem Korpus treffend benennt, einem bestimmten Typ von Vorkommen vorbehalten, wenn nämlich mit irgend-

1 Aachen, Bonn, Freiburg im Breisgau, Hamburg, Heidelberg, Mainz, Mannheim. 
wie signalisiert wird, dass eine Aussage unpräzis ist, dass sie (wahrscheinlich) nicht genau der Realität entspricht. Ein Grund für die Ungenauigkeit der Aussage kann dabei z.B. wie in (1) Unwissen über die tatsächlichen Gegebenheiten sein:

(1) Und beruflich, ähm, ich bin Lehrerin, ich arbeite voll. Ich hab nach dem ersten Kind ein halbes Jahr ausgesetzt [...] und ich hab, ja, eigentlich sofort wieder voll gearbeitet, weil ich an 'ner Grundschule bin und das ganz schön finde, wenn man 'ne eigene Klasse hat, dass man einfach da ist. Ich find das totaler Blödsinn, irgendwie nur, keine Ahnung, sieben Stunden da zu sein und andere machen ganz viel, aber es ist die eigene Klasse, man hat keine richtige Bindung zu den Kindern. (Charlotte)

Die Informantin, die als Lehrerin immer Vollzeit gearbeitet hat, ist sich nicht sicher, welche Stundenzahl einer Halb- oder Teilzeitstelle entspricht, weshalb sie den Vagheitsindikator irgendwie, begleitet von dem Ausdruck der Unwissenheit „keine Ahnung“, verwendet. Dem Adressaten wird so vermittelt, dass es sich bei „sieben Stunden“ nur um eine ungefähre Angabe, eine Vermutung seitens der Sprecherin handelt. Der Korpusauszug (2) ist insofern ähnlich gelagert, als es sich auch hier um eine ungefähre Angabe handelt, die allerdings diesmal in eine hypothetische, kontrafaktische Situation eingebettet ist: Die Informantin schätzt, dass sie, wenn ihre Examensnote nicht so gut gewesen wäre, ungefähr zwei Jahre lang an einer „schrecklichen Schule“ hätte ausharren müssen. Dass ihre Angaben nur approximativ (da hypothetisch) sind, wird auch durch „irgendwo“ und den Zusatz „oder so was“ ausgedrückt:

(2) Und wenn die [Note] gut genug ist, kriegt man eben auch sofort 'nen guten Job an 'ner guten Schule, also es lief alles total gut, ich musste nicht irgendwie zwei Jahre irgendwo in aller Mühe es an 'ner ganz schrecklichen Schule aushalten oder so was. Es war alles sehr... Ich hab auch Glück gehabt, glaub ich. (Charlotte)

Eine andere Erklärung für die Ungenauigkeit einer Aussage kann eine Gedächtnislücke oder, wie in (3), die Unmöglichkeit der Rekonstruktion einer (Anzahl von) Begebenheit(en) sein:

(3) Und der große [Bruder] ist jetzt fast vierzig, und hat immer noch keine Ausbildung abgeschlossen, und hat seit Jahren eine für alle Menschen, die ihn kennen, eindeutige schwere Depression, also inklusive: „Wie geht's Dir?“ „Ah, ich bin so müde, nee, ich hab schon wieder Halsschmerzen.“ Aber jeden Tag. Jeden Tag. Der ist nie ausgeschlafen, also der fühlt sich nie 
gut. Der hat immer was. Und dem geht's einfach total Scheiße, macht aber nichts dagegen, trotz irgendwie 'ner Million Gespräche, die wir mit ihm geführt haben. (Charlotte)

Auch hier ist die Ungenauigkeit der Angabe wieder doppelt markiert: einerseits durch das Vagheit anzeigende irgendwie, andererseits durch den unverkennbar hyperbolischen Ausdruck „,ner Million Gespräche“. Die Anzahl der Gespräche mit dem „großen Bruder“ wird auf diese Weise von der Sprecherin als so überdurchschnittlich hoch dargestellt, dass sie nicht mehr zählbar ist.

In den drei vorgestellten Korpusbelegen dieses Abschnitts erklärt sich die Markierung von Vagheit aus unterschiedlichen Gründen heraus: Ungewissheit bzgl. der genauen Verhältnisse (1), Spekulationen über eine hypothetische (da nie eingetroffene) Situation (2), rhetorisch-pragmatisches Mittel zum Ausdruck von Unzählbarkeit (3). Übrigens tritt irgendwie als Vagheitsindikator in unserem Korpus häufig, aber nicht ausschließlich, in Zusammenhang mit quantitativen Angaben auf. $\mathrm{Zu}$ einer anderen Vagheit indizierenden Verwendungsweise siehe weiter unten 2.5 .

\subsection{Formulierungsschwierigkeiten}

In einigen Fällen dient irgendwie als Signalisierung von Formulierungsschwierigkeiten. Hier wollen wir noch zwischen zwei Untertypen differenzieren. Diese Schwierigkeit entspricht nämlich manchmal einer reinen Wortsuche wie in (4) und (5), meistens handelt es sich aber um eine Schwierigkeit bei der Versprachlichung eines komplexen Sachverhalts, eines Gedankens, der noch im Entstehen ist, oder eines heiklen Themas wie in (6).

(4) Ja, der hatte mal unsere Schule mit Graffitis verunstaltet. Also Turnhalle war das gewesen. Und irgendwann sind sie ihm auf die Schliche gekommen, also, er ist erwischt worden, und die haben ja immer irgendwie ihre gleichen, na, wie heißt das? Ticks oder Gicks oder wie sich das schimpft, was die da machen. (Thorsten)

Die Problemsignalisierung wird hier noch prospektiv durch die Frage „na, wie heißt das?" verstärkt, und das Zögern beim Abrufen des richtigen Lexems wird zusätzlich retrospektiv durch den Ausdruck „oder wie sich das schimpft“ angezeigt. Irgendwie ist hier also ein Element einer Serie von sprachlichen Phänomenen, die Wortfindungsschwierigkeiten signalisieren. 
[(über Kleidung) Klassisch mögen Sie nicht?]

Klassisch find ich im Grunde genommen, so als Dauer, ich hab natürlich irgendwo ein Kostüm, weil man das ab und zu mal braucht, oder ich trag auch mal 'nen Hosenanzug, der irgendwo relativ klassisch ist, aber ansonsten ist das nicht meine Linie. Lieber was, ja, so kreativere Sachen, mit irgendwie, die irgend ... ich hab auch... deswegen trag ich die immer auch sehr lange. (Helga)

In diesem Interviewausschnitt wird die Wortsuche (und damit die Äußerung) sogar abgebrochen. In unserem Korpus erscheint irgendwie in solchen Kontexten fast ausschließlich als prospektive Problemsignalisierung, die also die Formulierungsschwierigkeit im Voraus ankündigt und auch als Verzögerungssignal fungiert.

Diese Verwendungsweise von irgendwie wird von Papantoniou (2010: 144) als ein untypischer Gebrauch von irgendwie und somit als ein Merkmal von Zweitsprachlichkeit behandelt. Unser Korpus zeigt aber Beispiele, wenn auch nicht viele, wo Muttersprachler irgendwie bei Störungen beim Abrufen eines Lexems benutzen.

Meistens aber liegt der Formulierungsschwierigkeit mehr als ein rein sprachlich bedingtes Problem zugrunde. Nach Papantoniou (2010) sind die häufigsten Kontexte, in denen irgendwie von Muttersprachlern benutzt wird, die Darstellung kognitiver Probleme (was unsere Korpusbelege nicht zu bestätigen scheinen) und die Versprachlichung von komplexen Sachverhalten. Anhand eines Beispiels aus einer Traumerzählung stellt er fest, dass der Sprecher irgendwie benutzt, „um seine Empfindungen als schwer formulierbar zu kennzeichnen“ (ebd.: 133). Ähnliche Beispiele finden wir in unserem Korpus:

[Hat Christopher seinen Vater gar nicht gekannt?]

Er war ja erst neun Monate alt. Und ich bin ... ich bin mir sicher, dass da irgendwie schon ... also das war irgendwie ganz ... wenn Georg nach Hause kam, dann war das natürlich Christopher, und der hat dann eben gelacht, und das war schon ganz toll, 'ne ganz intensive Beziehung, die die beiden zueinander hatten, aber da war natürlich auch, was weiß ich, in der ersten Zeit hab ich natürlich auch viel geweint, und er hat immer mitgeweint, aber ich wusste nicht, warum er jetzt auch weint. Aber, ich denke, er hat schon irgendwie so'n Empfinden, einfach, so'n Verlustempfinden schon gehabt. (Lea)

Hier versucht die Befragte, die schon mit zweiundzwanzig Witwe wurde, zu erklären, was ihr Sohn, der damals erst neun Monate alt war, beim Tod seines 
Vaters empfunden haben mag. Es ist zu erwarten, dass die Versprachlichung dieser traumatischen Situation der Befragten schwerfallen wird. Auffallend ist, dass die Befragte ihre Erklärungsversuche einleitet, indem sie behauptet, dass „[sie] [sich] sicher [ist]“, um dann eher ihre Unsicherheit (unter anderen sprachlichen Signalen) durch das gehäufte Auftreten von irgendwie auszudrücken. Zwar ist sie sich sicher, dass ihr Sohn unter einem Verlustgefühl gelitten hat, wobei ihr aber gleichzeitig klar ist, dass bei einem neun Monate alten Baby dieses Verlustempfinden nicht rational bewusst gewesen sein kann. Sie kann nicht versprachlichen, in welcher Form sich bei einem so jungen Kind ein Verlustempfinden manifestiert.

Ein anderer, aber ähnlicher Typ von Versprachlichungsschwierigkeiten findet sich bei Gülich/Furchner (2002). In dieser Studie stammen die Beispiele aus Gesprächen zwischen Ärzten und Patienten, die unter epileptischen Auren leiden. Die Patienten haben Schwierigkeiten, ihre Symptome zu versprachlichen und benutzen u.a. irgendwie, um diese Unbeschreibbarkeit $\mathrm{zu}$ signalisieren: „[Die Schwierigkeit, das von den Patienten Erlebte zu beschreiben], manifestiert sich neben der Feststellung von Unvertrautheit des Phänomens [...] auch in Vagheitsindikatoren (irgendwie) und zahlreichen Verzögerungen (ähm, Pausen, Dehnungen).“ (ebd.: 165).

Formulierungsschwierigkeiten (insbesondere bei der Versprachlichung eines komplexen bzw. heiklen Sachverhalts) stellen also eine übliche Verwendungsmodalität von irgendwie dar.

\subsection{Unerklärbarkeitsmarker}

Eine weitere Verwendungsmodalität von irgendwie kann anhand folgender Korpussequenzen veranschaulicht werden:

(7) Aber ich mag das so, genauso, mit dem wahnsinnig vielen Glas, wir haben ein Phantomhaus vorher gesehen, mal irgendwann, im Schöner Wohnen, als wir noch ganz jung waren, und das haben wir gesagt: „Wenn wir mal bauen, so soll's eigentlich ungefähr aussehen.“ Und dann haben wir das Bild weggelegt, und haben's nicht mehr gefunden. Und irgendwie war es weg. Und zehn Jahre später, da war das Haus dann gebaut, war's wieder, haben wir's gefunden, und war faszinierend: Es gibt genau, vom Charakter her, überhaupt nicht vom Grundriss und gar nicht von, sondern allein vom Charakter her genau das ab, was wir damals gerne haben wollten. (Helga) 
Und da ist jetzt im Prinzip so noch seine Mutter und seine Großmutter übrig. Er hat noch 'nen Bruder, der ist Psychoanalytiker in Berlin, aber wir sehen uns so alle zwei Jahre mal. Da ist leider irgendwie wenig Kontakt, obwohl wir uns auch sehr mögen, aber, das ist irgendwie... das würd ich auch nicht als Familie bezeichnen. Also nicht meine Familie. (Lea)

Irgendwie erscheint in beiden Ausschnitten im Zusammenhang mit einer Feststellung und drückt aus, dass diese sich nicht erklären lässt. In (7) hat die Befragte keine Erklärung dafür, warum das Bild auf einmal nicht mehr zu finden war (aber nicht ganz verschwunden war, denn es ist nach zehn Jahren erneut aufgetaucht). In (8) wird die Inkohärenz der Situation durch obwohl deutlich markiert: Diese Menschen sehen sich wenig, obwohl sie sich nahestehen. Es gibt also keinen plausiblen Grund dafür, dass so wenig Kontakt besteht. In diesen Kontexten bringt irgendwie die Unerklärbarkeit zum Ausdruck.

Unseres Erachtens könnte diese Interpretation das „Krankenwagen“-Beispiel $^{2}$ von Günthner/König (2015) aus einem anderen Blickwinkel beleuchten:

Bei einem Radio-phone-in berichtet der Anrufer Tim dem Moderator Danni von seiner Hilflosigkeit, als er seine Mutter tot aufgefunden hat.

D: Hast du denn irgendwie einen Rettungswagen...die Polizei oder irgendeinen Freund oder Verwandten angerufen?

T: Ja auf den auf ' $n$ Krankenwagen bin ich gar nicht darauf gekommen irgendwie;' ich hatte erst versucht meinen Vater anzurufen, der den dann 'ne Stunde mit dem Auto entfernt war. (ebd.: 264)

Die Autorinnen interpretieren gar nicht in Tims Äußerung „[ich] bin gar nicht darauf gekommen“ als eine „extreme case formulation“ und irgendwie folglich als eine retrospektive abschwächende Korrektur: „Entsprechend fügt Tim noch in derselben Turnkonstruktionseinheit ein ausgeklammertes, unakzentuiertes irgendwie an, mit dem er die zuvor getätigte hyperbolische Äußerung nachträglich abschwächt.“ (ebd.: 264). Wir sehen darin weniger eine extreme Formulierung als eine Feststellung, durch die der Sprecher ausdrückt, dass ihm seine eigene Verhaltensweise unerklärlich bleibt: Tim fällt auf, dass er tatsächlich nicht daran gedacht hat, einen Krankenwagen anzurufen. Ihm wird bewusst, dass es in seiner Situation eine erwartbare Reaktion gewesen wäre, kann sich aber nicht erklären, wieso er anders gehandelt hat. In diesem Fall kann irgendwie zwar auch

2 Das hier in literarischer Umschrift wiedergegeben wird.

3 Von uns hervorgehoben. 
abschwächend wirken, aber es bringt unseres Erachtens ebenfalls diese Unerklärbarkeit zum Ausdruck.

\subsection{Abschwächung}

Für Thaler (2008), in Anschluss an Fraser (1980), ist irgendwie eines der möglichen Mittel zur Abschwächung (,mitigation“) sprachlicher Äußerungen. Abschwächung (wie auch ihr Gegenstück Verschärfung) gehört laut Thaler zu den geläufigen, ja sogar universellen konversationellen Strategien, derer sich pragmatisch kompetente Sprecher bedienen. Fraser bemerkt, dass Abschwächung auf zwei verschiedene Arten motiviert sein kann: „the motivation to mitigate appears to fall into two, not always unrelated, categories: self-serving and altruistic“ (1980: 344). In beiden Fällen geht es um Abwendung oder zumindest um Reduzierung des Ausmaßes einer potenziellen Gesichtsbedrohung: der des Sprechers selbst oder der des Adressaten. Je nach Art der Kommunikationssituation und Rollenverteilung ist von Seiten der Sprecher also eher mit eigennützig oder mit altruistisch motivierter Abschwächung zu rechnen. So zeigen z.B. MeyerHermann/Weingarten (1982) mittels eines Korpus von Gruppentherapiegesprächen, wie irgendwie von den Therapeuten im Verlauf von Therapien (altruistisch) eingesetzt wird, um Äußerungen von Patienten abschwächend zu reformulieren und damit einen möglichen Gesichtsverlust bei anderen Teilnehmer/innen der Therapiegespräche zu vermeiden bzw. zu reduzieren.

Unsere eigenen Korpusdaten beruhen auf Interviews, also auf einer Art von Kommunikationssituation, die eindeutig gesichtsbedrohender für den Informanten/die Informantin als für den Interviewer/die Interviewerin ist. So bemerken wir auch in (9) zwei Vorkommen von abschwächendem irgendwie, die der Sprecher zur eigenen Gesichtswahrung einsetzt:

Dann war er also eine Woche in dem Heim, eine Woche bei uns, eine Woche bei seiner Mutter, dann wieder eine Woche in dem Heim, dann wieder ... Und das war dann schon auch intensiver. Und mittlerweile ertapp ich mich selbst dabei, dass, wenn ich sag, ich hab ... ich bin ... ich hab also drei Kinder, dass ich irgendwie denke, na, der Mathias ist irgendwie gar nicht mehr so richtig dabei. Weil er einfach nur noch selten da ist. (Frank)

Der Sprecher, der aus einer früheren Beziehung ein behindertes, in einem Heim lebendes Kind (Mathias) hat, berichtet über den Besuchsrhythmus, der zum Zeitpunkt des Interviews auf nur ein Wochenende alle sechs Wochen reduziert ist. Dieser stark eingeschränkte Kontakt führt dazu, dass Frank zuweilen seinen 
Sohn als nicht mehr zur Familie gehörig empfindet - ein Gedanke, der bei ihm Schuldgefühle auslöst (,ertapp ich mich selbst dabei“). Einen solchen Gedanken zu äußern birgt die Gefahr einer negativen Einschätzung durch den Adressaten/ die Adressatin (hier die Interviewerin). So wird die Äußerung auch nicht nur durch zweimaliges Verwenden von irgendwie abgeschwächt, sondern der Sachverhalt zudem mit einer durch „,weil“ eingeleiteten Nachfolgeäußerung begründet. Die Präsenz der Abtönungspartikel „einfach“, die die Lage wie eine Fatalität hinstellt, wirkt zusätzlich rechtfertigend.

Die Verwendung von abschwächendem irgendwie in (10) ist interessant, da es sich hier um die Wiedergabe einer wörtlichen Rede handelt:

Und ich hab dann halt auch gemerkt, das wird immer schwieriger, irgendwie so Grenzen zu ziehen, und zu sagen: „Das ist ganz nett, dass du uns jetzt besuchst, aber das ist irgendwie ... du musst halt irgendwie heut' Abend auch wieder gehen.“ [...] Also, er hat schon versucht, eigentlich, da so 'ne Rolle einzunehmen, die ich aber überhaupt gar nicht wollte. (Lea)

Die Informantin erzählt in dieser Passage von einer Situation (sie hatte einige Monate vorher ein Kind von einem Mann bekommen, mit dem sie nicht zusammenleben wollte, und mit dem sie keinerlei Zukunftspläne hegte), die beinahe zwanzig Jahre zurückliegt. Es erscheint demnach ausgeschlossen, dass es sich bei der wörtlichen Rede um den getreuen Wortlaut einer tatsächlich so formulierten Äußerung handelt. Wir haben es also mit der Konstruktion eines Mini-Szenarios zu tun, bei der die Sprecherin durch den Einsatz von irgendwie, gekoppelt mit dem Abbruch und der Wiederaufnahme der Äußerung, einen doppelt positiven Effekt erzielt: Zum einen erscheint die direkte Rede dadurch authentischer (der zweifache Gebrauch des Abschwächungsmarkers spiegelt die Peinlichkeit der Situation, in der sie den Vater ihres Kindes des Hauses verweist, effizient wider), zum anderen stellt sie sich selbst als Sprecherin dar, die dem Adressaten gegenüber gesichtsschonend, also altruistisch, agiert.

Um altruistisch motivierte Abschwächung geht es auch in (11), einem eher untypischen Beispiel, da es hier die Interviewte ist, die eine gesichtsbedrohende Frage an die Interviewerin mithilfe von irgendwie abzuschwächen sucht:

Ja müssen Sie jetzt nix irgendwie aufschreiben? (Mechthild)

Tatsächlich stellt hier die Informantin (welche das vor ihr liegende Aufnahmegerät nicht als solches identifiziert hat) fest, dass sich die Interviewerin, nachdem das Interview schon vor einigen Minuten begonnen und sie schon auf mehrere Fragen geantwortet hat, keinerlei Notizen macht. Sich danach zu 
erkundigen, ob die Interviewerin „nix aufschreiben [müsse]“, kommt einer Gesichtsbedrohung gleich, da letztere damit als potenziell unprofessionell oder unseriös denunziert werden könnte. Durch die Frageform der Äußerung sowie durch das abschwächende irgendwie signalisiert aber die Sprecherin gesichtsschonend, dass sie Optionen für denkbar hält, die das Verhalten der Interviewerin legitimieren.

\subsection{Exemplifizierendes irgendwie}

Bei einer Reihe der Vorkommen in unserem Korpus dient irgendwie zur Exemplifizierung und ist in diesen Fällen folglich durch zum Beispiel austauschbar. Der Beispielcharakter des durch irgendwie eingeführten Sachverhalts ist dabei zusätzlich daran erkennbar, dass im Kotext auf Alternativen zu diesem Sachverhalt hingewiesen wird. Dies geschieht in (12) mithilfe von „oder so“ nur implizit, da es hier dem Adressaten überlassen bleibt, sich alternative Situationen zu „essen [sein]“ (d.h. zu einem Restaurantbesuch mit Freunden) vorzustellen:

(12) Aber wenn er [= mein Mann] ein Thema hat, das ihn interessiert, oder wo er gerade irgendwie dran ... gedanklich beschäftigt ist, dann kann der auch ganz viel erzählen. Und vor allem in Gesellschaft. Also, wenn wir mit Freunden irgendwie essen sind oder so, dann kann der sehr unterhaltsam sein. (Charlotte)

In (13) und (14) werden Alternativen auch explizit gemacht:

(13) Und auf einmal melden sich Leute halt bei meinen Eltern und sagen: „Wie kann eure Tochter jetzt mit so einem unterwegs sein?" Wo ich so gar nichts mit anfangen konnte. Also er war ja nicht irgendwie aus dem Gefängnis oder rauschgiftsüchtig oder von der Straße aufgelesen oder irgendwie so. (Juliet)

An der parataktischen Aufzählung verschiedener Möglichkeiten (,aus dem Gefängnis“, „rauschgiftsüchtig“, „von der Straße aufgelesen“) wird deutlich, dass es sich hier um mögliche Beispiele handelt, wobei die Sprecherin die Liste der Optionen durch abschließendes irgendwie so als offen kennzeichnet. Bezeichnenderweise findet sich exemplifizierendes irgendwie in unserem Korpus in vielen Fällen von (oder) so begleitet. Auch in (14) werden Alternativen ausdrücklich genannt („ein Bier oder eine Chipstüte“), und auch hier wird der Beispielcharakter der Aufzählung durch abschließendes „oder sonst was“ rückwirkend noch- 
mals bestätigt. Zudem führt der Sprecher mit „,sagen wir mal“ die Situation insgesamt als hypothetisch und beispielhaft ein:

(14) Ich bin jetzt nicht einer, der gerne aufräumt oder gern putzt, ich bin dann eher jemand, der sagt, ich schau, dass ich erst gar keine Unordnung aufkommen lasse, das ist wirklich so. Also das heißt, wenn ich jetzt wirklich, sagen wir mal, ich sitze vorm Fernseher und hab jetzt da irgendwie ein Bier oder eine Chipstüte oder sonst was, und ich stehe dann auf, dass ich das dann gleich mitnehme, und wenn ich eh in die Küche laufe, dass das dann gleich fort ist. (Karl-Heinz)

Ein - keinesfalls seltener - Sonderfall von exemplifizierendem irgendwie ist dessen Verwendung als einführendes Element einer Redewiedergabe. In (15) äußert sich der Sprecher zu der Schwierigkeit, zutreffende Benennungen für die verschiedenen Mitglieder einer Patchworkfamilie $\mathrm{zu}$ finden, und illustriert dies anhand einer (vermeintlichen) Redewiedergabe, die er durch irgendwie einführt und somit als exemplifizierend kennzeichnet. Wiederum wird der Beispielcharakter des Sachverhalts (hier in Form einer direkten Rede) durch nachgeschobenes „oder irgendwie so“ retrospektiv bestärkt:

Und ich hab jetzt auch, jetzt in meinem Job hab ich das als ... wie soll ich sagen? recht praktikable Lösung gesehen, weil ich derzeit auch einige Kinder hab in meiner Klasse, die die Situation haben. Dass sie irgendwo einen leiblichen Vater haben, aber mit dem ... wo der Freund, der neue Freund der Mutter, oder der neue Mann, auch lebt mit der Mutter und auch im Grunde schon ... Dann ging's immer darum, dass es Unklarheiten dann gab, irgendwie: „Dein Papa“ „Nee, das ist aber nicht mein Papa“, oder irgendwie so. (Frank)

Dass die immer noch verbreitete Auffassung, derzufolge Redewiedergabe in direkter Form dazu diene, tatsächliche vergangene Äußerungen zu zitieren, in sehr vielen Fällen unzutreffend ist, wurde mehrfach belegt (siehe u.v.a. Aufray 2010; Günthner 2005). Schon Ende der 1980er Jahre hatte es Tannen (1989: 101) auf die knappe Formel gebracht: „Much of what appears in discourse as dialogue, or 'reported speech', was never uttered by anyone else in any form. “ Tatsächlich handelt es sich sehr oft um eine Inszenierung, ein In-Szene-Setzen, manchmal einer einmaligen Situation, die tatsächlich stattgefunden hat, häufig aber einer typischen Situation, die immer wieder so, oder so ähnlich, stattfindet bzw. so stattfinden könnte. Das heißt, Situationen werden mittels direkter Rede exemplifiziert, wie es auch an (16) leicht erkennbar wird, wo die Sprecherin den 
Ablauf eines typischen Nachmittags, an dem sie ihre Tochter aus der Kinderkrippe abholt, anhand von direkter Rede, die sie mit irgendwie einführt, in Szene setzt:

(16) Weil man nachmittags halt schon zu fünfzig Prozent auf'm Spielplatz 'rumhängt, weil die Kinder sich's halt wünschen, und meistens ist es so nach der Krippe, dass um drei so'n Pulk, so 'ne Traube Mütter vor der Krippe steht, und dann ist irgendwie: „Was macht ihr jetzt, was macht ihr jetzt?“ „Ja, wir gehen auf den Wasserspielplatz.“ „Ach ja stimmt, ist schön warm.“ „Habt ihr Handtücher mit?“ „Ja klar.“ „Ja, wir kommen mit.“Ja so. (Charlotte)

Allerdings muss redeeinführendes irgendwie nicht immer nur exemplifizierend sein. So signalisiert in (17) die Sprecherin durch die Verwendung des Lexems, dass sie sich des genauen Wortlauts der (in diesem Fall höchstwahrscheinlich tatsächlich geäußerten) wiedergegebenen direkten Rede nicht sicher ist. Dieses irgendwie gehört also prioritär in die bereits behandelte Kategorie der Vagheitsindikatoren (siehe oben 2.1):

(17) Also, wir sind sehr, wir sind sehr, halt sehr ehrlich und sehr offen und reden auch über so ganz grundlegende Sachen. Wo bin ich jetzt grad in meinem Leben und ... Ohne dass wir uns das so vornehmen, das kommt einfach so. Auch über Schule, weil das halt beide verbindet, weil sie auch Lehrerin ist. Und von Christine erzähl ich mal so 'ne Anekdote, ja, wenn sie grad, heute Morgen hat sie gesagt irgendwie: „Ich glaub Mama, ich mag den Wind doch nicht so gerne.“ (Charlotte)

Exemplifizierendes irgendwie scheint auf den ersten Blick von den anderen Verwendungsweisen des Lexems semantisch abzuweichen. Dennoch findet sich bei genauerem Hinsehen auch hier die Grundbedeutung des Sich-nicht-festlegenKönnens (wie etwa im Falle von Formulierungsschwierigkeiten oder Unerklärbarkeit eines Sachverhalts) oder -Wollens (wie z.B. bei Abschwächung oder Exemplifizierung) seitens des Sprechers: Der Unbestimmtheits- oder Unbestimmbarkeitscharakter bleibt letztlich allen Verwendungsweisen von irgendwie gemeinsam. Über diese konstante Grundbedeutung hinaus variieren die Verwendungsweisen von irgendwie auf pragmatischer Ebene: Wie unsere Korpusbelege zeigen, haben Sprecher unterschiedliche Gründe, aus denen sie Unbestimmtheit ausdrücken. Letztere sind nicht immer eindeutig zu isolieren. So wird beispielsweise an Korpusauszug (17), wo sich der Ausdruck von Vagheit und von Exemplifizierung zu überschneiden scheinen, eine gewisse Durchlässigkeit der hier vorgestellten Verwendungskategorien deutlich. 


\section{Schlussbemerkungen}

Die Analyse unseres Korpus hat ermöglicht, eine Reihe verschiedener Verwendungsmodalitäten von irgendwie herauszuarbeiten, während andere Studien nachgewiesen haben, dass das Auftreten einer bestimmten Gebrauchsweise von irgendwie sprechsituationsgebunden sein kann. Meyer-Hermann/Weingarten (1982) zeigen beispielsweise, wie irgendwie von Therapeuten im Rahmen von Gruppentherapiegesprächen gebraucht wird, um Patientenäußerungen abschwächend zu reformulieren und damit einen möglichen Gesichtsverlust bei einem anderen Teilnehmer zu vermeiden bzw. zu reduzieren. In dem von Gülich/Furchner (2002) ausgewerteten Korpus wird das Auftreten von irgendwie als eines der Mittel zur sprachlichen Markierung der Unbeschreibbarkeit von Krankheitssymptomen analysiert. Es ist daher anzunehmen, dass die Berücksichtigung weiterer Typen von Korpora das Spektrum unserer vorläufigen, auf semantischen und pragmatischen Kriterien beruhenden Klassifizierung erweitern würde.

Die Verwendung von irgendwie erweist sich außerdem nicht nur als (potenziell) sprechsituationsabhängig, sondern auch als sprechergebunden: So betreffen in unserem Korpus 96 von insgesamt 360 Vorkommen von irgendwie die gleiche Sprecherin (Lea), während neun Informant/innen während des gesamten Interviews nur ein oder zwei Mal auf das Lexem zurückgreifen. Bei lediglich einer Informantin kommt irgendwie überhaupt nicht vor. Diese Diskrepanz könnte damit zusammenhängen, dass die Interviewsituation von den Sprecher/innen unterschiedlich eingeschätzt und erlebt wird. Um diese Hypothese zu überprüfen, müssten die gleichen Sprecher/innen in verschiedenen Kommunikationssituationen beobachtet werden.

$\mathrm{Zu}$ klären bliebe auch, ob die syntaktische Position des Lexems Einfluss auf dessen pragma-semantische Funktion nimmt. Einen ersten wichtigen Schritt in diese Richtung haben Günthner/König (2015) in ihrer Untersuchung zu irgendwie im Nachfeld unternommen.

\section{Literatur}

Aufray, Antoine (2010): Weil da so ,ja kommen Sie mal morgen': Étude syntaxique et discursive du discours rapporté en allemand oral contemporain. Paris: Université Paris-Sorbonne IV. [Unveröffentlichte Dissertation].

Duden (2016): Duden 4: Die Grammatik. 9., vollst. überarb. u. aktual. Aufl. Berlin: Dudenverlag. Fraser, Bruce (1980): Conversational mitigation. In: Journal of Pragmatics 4, 4. 341-350.

Gülich, Elisabeth/Furchner, Ingrid (2002): Die Beschreibung von Unbeschreibbarem. Eine konversationsanalytische Annäherung an Gespräche mit Anfallskranken. In: Keim, 
Inken/Schütte, Wilfried (Hgg.): Soziale Welten und kommunikative Stile. Tübingen: Narr. 161-185.

Günthner, Susanne (2005): Fremde Rede im Diskurs. Formen und Funktionen der Polyphonie in alläglichen Redewiedergaben. In: Assmann, Aleida/Gaier, Ulrich/Trommsdorff, Gisela (Hgg.): Zwischen Literatur und Anthropologie. Diskurse, Medien, Performanzen. Tübingen: Narr. 339-359.

Günthner, Susanne/König, Katharina (2015): Temporalität und Dialogizität als interaktive Faktoren der Nachfeldpositionierung - , irgendwie‘ im gesprochenen Deutsch. In: Vinckel-Roisin, Hélène (Hg.): Das Nachfeld im Deutschen. Theorie und Empirie. (= Reihe Germanistische Linguistik 303). Berlin/Boston: De Gruyter. 255-278.

Jabs, Britta (2012): Styles de vie et façon de parler. Etude de quelques formes de discours dans différents milieux socio-culturels en Allemagne contemporaine. Paris: Université Paris-Sorbonne IV. [Unveröffentlichte Dissertation].

Meyer-Hermann, Reinhard/Weingarten, Rüdiger (1982): Zur Interpretation und interaktiven Funktion von Abschwächungen in Therapiegesprächen. In: Detering, Klaus/SchmidtRadefeld, Jürgen/Sucharowski, Wolfgang (Hgg.): Sprache erkennen und verstehen. Akten des 16. Linguistischen Kolloquiums. Tübingen: Niemeyer. 242-252.

Papantoniou, Theodoros (2010): Zur zweitsprachlichen Spezifik von Signalisierungsmitteln bei Sprachproduktionsproblemen: Die Verwendung des Heckenausdrucks ,irgendwie“ in der mündlichen Kommunikation. In: Krause, Wolf-Dieter (Hg.): Das Fremde und der Text. Fremdsprachige Kommunikation und ihre Ergebnisse. Potsdam: Universitätsverlag Potsdam. 119-152.

Schanen, François/Confais, Jean-Paul (2001): Grammaire de l'allemand. Formes et fonctions. Paris: Armand Colin.

Schwitalla, Johannes (1997): Gesprochenes Deutsch. Eine Einführung. Berlin: Schmidt. Tannen, Deborah (1989): Talking voices: Repetition, dialogue, and imagery in conversational discourse. (= Studies in Interactional Sociolinguistics 6). Cambridge, UK: Cambridge University Press.

Thaler, Verena (2008): L'enjeu de la compétence pragmatique en langue étrangère. Une étude sur les procédés de mitigation en allemand L1 et L2. In: Revue Canadienne de Linguistique Appliquée 11, 3. 193-214. 